\title{
Atomic Multiplet and Charge Transfer Effects in the Resonant Inelastic X-Ray Scattering (RIXS) Spectra at the Nickel $\mathrm{L}_{2,3}$ Edge of $\mathrm{NiF}_{2}$
}

\author{
J JIMÉNEZ-MIER, ${ }^{1, *}$ P OLALDE-VELASCO,${ }^{2}$ P DE LA MORA, ${ }^{3}$ W-L YANG,${ }^{4}$ \\ AND J DENLINGER ${ }^{4}$ \\ ${ }^{1}$ Instituto de Ciencias Nucleares, UNAM, 04510 Ciudad de México, México \\ ${ }^{2}$ Instituto de Física, Benemérita Universidad Autónoma de Puebla, Puebla, A. Postal \\ J-48 Puebla, Puebla 72750, Mexico \\ ${ }^{3}$ Facultad de Ciencias, UNAM, 04510 Ciudad de México, México \\ ${ }^{4}$ The Advanced Light Source, Lawrence Berkeley Laboratory, Berkeley, CA 94720, USA \\ *Email: jimenez@nucleares.unam.mx
}

Published online: August 07, 2017

The Author(s) 2017. This article is published with open access at www .chitkara.edu. in/publications

\begin{abstract}
Resonant inelastic x-ray scattering (RIXS) is used to study the electronic structure of $\mathrm{NiF}_{2}$, which is the most ionic of the nickel compounds. RIXS can be viewed as a coherent two-steps process involving the absorption and the emission of x-rays. The soft X-ray absorption spectrum (XAS) at the metal $\mathrm{L}_{2,3}$ edge indicate the importance of atomic multiplet effects. RIXS spectra at $\mathrm{L}_{2,3}$ contain clearly defined emission peaks corresponding to d-excited states of $\mathrm{Ni}^{2+}$ at energies few eV below the elastic emission, which is strongly suppressed. These results are confirmed by atomic multiplet calculations using the Kramers-Heisenberg formula for RIXS processes. For larger energy losses, the emission spectra have a broad charge-transfer peak that results from the decay of hybridized $\mathrm{Ni}(3 \mathrm{~d})-\mathrm{F}(2 \mathrm{p})$ valence states. This is confirmed by comparison of the absorption and emission spectra recorded at the nickel $\mathrm{L}$ and fluorine $\mathrm{K}$ edges with F p and Ni d partial density of states using LDA + U calculations.
\end{abstract}

Keywords: Core-level spectroscopies. RIXS, Nickel difluoride, Electronic structure

\section{INTRODUCTION}

Transition metal (TM) compounds play a major role in condensed matter physics and materials science. A complex interplay between the charge, orbital,

Journal of Nuclear

Physics, Material Sciences, Radiation and

Applications

Vol-5 No-1

August 2017 pp. 1-13 
Jiménez-Mier, J Olalde-Velasco, $\mathrm{P}$ de la Mora, $\mathrm{P}$ Yang, W -L Denlinger, $\mathrm{J}$

spin and lattice degrees of freedom gives birth to emergent phenomena like high temperature superconductivity (HTSC), colossal/giant magneto resistivity (C/GMR) and thermoelectricity in copper, manganese and cobalt oxides, respectively [5]. Understanding the origin of these emergent phenomena has prompted theoretical and experimental research which points to the crucial role of $3 \mathrm{~d}$ electronic correlations. Indeed, the insulating Mott state and its related metal to insulator transitions (MITs) are a living classic in contemporary condensed matter physics [12]. The advance in the understanding of these phenomena requires combined efforts between theory and experiment. Therefore, systematic investigations on model systems are crucial to establish solid foundations in this regard. For example, the simplest $3 \mathrm{~d}$ transition metal oxides (TMOs), e.g. $\mathrm{MnO}, \mathrm{CoO}$, and $\mathrm{NiO}$ have allowed us to reach the current understanding of the electronic structure of these materials and their insulating bandgap either as Mott-Hubbard or as charge-transfer (CT) insulators, as stated by the Zaanen-Sawatzky-Allen (ZSA) diagram [25]. However, it took more than two decades to have a direct experimental proof of the ZSA theory and it came from a combined experiment of X-ray absorption (XAS) and emission (XES) spectroscopies on the most ionic TM compounds, the difluorides TMF2 (Cr-Zn) at the $\mathrm{TM} \mathrm{L}_{2}, 3$ and $\mathrm{F} \mathrm{K}$ edges [18].

Among the simplest TMOs, $\mathrm{NiO}$ is the test ground of the interplay between theory and experiment. Although there is a comparative study between $\mathrm{NiO}$ and $\mathrm{NiF}_{2}[15]$, overall the latter has been marginally studied. However several parallelisms can be established: both solids are antiferromagnetic insulators with the $\mathrm{Ni}^{2+}$ ions at the center of a perfect octahedra (Oh symmetry in $\mathrm{NiO}$ ) and slightly distorted one (D4h symmetry in $\mathrm{NiF}_{2}$ ) surrounded by six ligands $(\mathrm{O}$ or $\mathrm{F})$. Interestingly, one important difference, consequence of the strong ionicity in $\mathrm{NiF}_{2}$, is the fact that according to ZSA theory $\mathrm{NiO}$ is in the intermediate region between Mott-Hubbard and charge-transfer insulator regimes while $\mathrm{NiF}_{2}$ is a clear Mott-Hubbard insulator [18]. This indicates that replacing $\mathrm{O}$ by $\mathrm{F}$ induces profound changes in the electronic structure of NiF2. These changes naturally should be reflected in the absorption (XAS), emission (XES), photoelectron (XPS) and resonant inelastic scattering (RIXS) $\mathrm{X}$-ray spectra. For instance, the $\mathrm{XAS}$ spectra of $\mathrm{Ni}^{2+}$ in $\mathrm{NiO}$ and $\mathrm{NiF}_{2}$ were analyzed by van der Laan et al. [15] and Olalde et al. [19] using, respectively, many-body calculations including charge transfer (CT) effects and ligand field atomic multiplet calculations. From the analysis it was shown that CT effects were more important to describe completely all the features in the $\mathrm{NiL}_{2,3} \mathrm{XAS}$ spectrum in $\mathrm{NiO}$ as compared to $\mathrm{NiF}_{2}$. On the other hand, in XES spectroscopy, $\mathrm{CT}$ effects, from ligand ( $\mathrm{F}$ or $\mathrm{O}$ ) to metal and vice versa were also observed in $\mathrm{TMF}_{2}$ including $\mathrm{NiF}_{2}[18]$. At the $\mathrm{Ni} \mathrm{L}_{2,3}$ edges, RIXS grants access to neutral 
optically forbidden Ni dd and F to Ni CT excitations. In RIXS, the ligand to metal CT effects at the $\mathrm{Ni}_{3}$ [7] and $\mathrm{M}_{2,3}$ [3] edges have also been observed by RIXS in $\mathrm{NiO}$ and $\mathrm{NiCl}_{2}$, but to the best of our knowledge not in $\mathrm{NiF}_{2}$. Thus, a systematic RIXS study at the $\mathrm{NiL}_{2,3}$ edges in $\mathrm{NiF}_{2}$ should in principle, in combination with all the previous spectroscopic results, provide a more complete picture of the electronic structure of the material.

Theoretically, the electronic band structure of $\mathrm{NiF}_{2}$ has been studied using the full potential linearized augmented plane wave (LAPW) method, with the exchange and correlation effects treated in the local spin-density approximation (LSDA) [6]. This calculation correctly predicts the insulating ground state in NiF2 but the bandgap is largely underestimated as compared to the experimental determination obtained from soft X-ray spectroscopies [18].

In practical terms, $\mathrm{NiF}_{2}$ has recently received attention for various reasons. It was used to produce 3-D nanoporous flexible electrodes for commercial applications [24]. It shows potential as a cathode material for rechargeable $\mathrm{Li}$ ion batteries $[16,26]$ which in a $\mathrm{NiO}$-doped $\mathrm{NiF}_{2}-\mathrm{C}$ version of the previous cathodes was investigated by hard X-ray absorption spectroscopy [17]. It has also been used as catalyst for the dehydrogenation of ammonia borane [23], a proposed material for hydrogen storage. Therefore, from the fundamental physics point of view as well as for the applicability of $\mathrm{NiF}_{2}$ as a practical material in energy storage and catalysis, it is crucial to pin down the energy levels of $\mathrm{Ni}$ and $\mathrm{F}$ in this material.

\section{X-RAY ABSORPTION, X-RAY EMISSION AND RIXS SPECTROSCOPIES}

Soft x-ray spectroscopies are very well suited to study the electronic structure of transition metal compounds [9]. In the case of nickel fluoride, the fluorine $\mathrm{K}$-edge is at $685 \mathrm{eV}$ and the nickel $\mathrm{L}_{2,3}$ edges are at $850 \mathrm{eV}\left(\mathrm{L}_{3}\right)$ and 867 $\mathrm{eV}\left(\mathrm{L}_{2}\right)$. In x-ray absorption spectroscopy (XAS) a photon is used to excite a core electron into the conduction band. The core hole can then be filled by an electromagnetic transition involving an electron in the valence band, resulting in x-ray emission spectra (XES). Resonant inelastic x-ray scattering (RIXS) is a coherent second order process in which an incoming photon of energy $h v_{1}$ is scattered, resulting in an outgoing photon with energy $h v_{2}$. The energy lost in RIXS, given by $h v_{1}-h v_{2}$, is transferred to the sample. Therefore, RIXS spectra plotted as functions of the energy loss give information about excited states of the compound that is studied.

$\mathrm{X}$-Ray absorption and emission are local processes at the atomic level. Fluorine XAS and XES spectra probe conduction and valence states projected
Atomic Multiplet and Charge

Transfer Effects in the Resonant Inelastic X-Ray Scattering (RIXS) Spectra at the Nickel $\mathrm{L}_{2,3}$ Edge of $\mathrm{NiF}_{2}$ 
Jiménez-Mier, J Olalde-Velasco, P de la Mora, $\mathrm{P}$ Yang, W -L Denlinger, $\mathrm{J}$ at this atom site. By the same token, nickel XAS and XES spectra give information about unoccupied and occupied states at the metal. Furthermore, the transitions are governed by the electric-dipole selection rules, and therefore the incoming and outgoing $\mathrm{x}$-ray photons give information about conduction and valence states of specific orbital symmetry. In fluorine XAS a 1s electron is excited to $2 \mathrm{p}$ conduction states, and the 1 s hole is filled by valence $2 p$ electrons. In nickel XAS a 2 p electron is promoted to conduction states with $3 d$ symmetry. In RIXS at the nickel $\mathrm{L}_{2,3}$ edges, the metal emission that follows resonant excitation of a $2 p$ electron produces d-excited states of the ground configuration.

\section{THEORY}

\section{A. Atomic multiplet calculations}

A theoretical description of the electronic structure of transition metal compounds is complicated by the fact that the $3 \mathrm{~d}$ orbitals are spatially localized like those of shallow core-orbitals, but at the same time their energies are low, making them to participate in chemical bonding. The atomic multiplet approach $[10,11]$ begins with a description of the nickel electronic structure at the freeion level. The ground state configuration for $\mathrm{Ni}^{2+}$ is $3 \mathrm{~d}^{8}$ which is excited by the incoming photon to states in the $2 p^{5} 3 \mathrm{~d}^{9}$ configuration. The calculation includes the electron-electron Coulomb interaction through the $F$ and $G$ Slater integrals, and also the $\zeta 2 p$ and $\zeta_{3 \mathrm{~d}}$ spin-orbit interaction parameters. These parameters are obtained directly from a Hartree-Fock calculation for the freeion [4]. The two $3 d$ holes in the ground configuration result in a ${ }^{3} F$ ground term. Including spin-orbit interaction of the $3 \mathrm{~d}$ subshell gives a ground total angular momentum of $J=4$. At room temperature only this ${ }^{3} \mathrm{~F} 4$ ground state can be excited by electric dipole transitions into states of the $2 p^{5} 3 \mathrm{~d}^{9}$ configuration. All other terms will not be excited, but they can be produced as result of x-ray emission. Decay into these $3 d$ excited states plays a very important role in the RIXS process. Besides the ${ }^{3} F$, the excited terms in the $3 \mathrm{~d}^{8}$ configuration are the singlets and triplets ${ }^{1} D,{ }^{3} \mathrm{P},{ }^{1} G$ and ${ }^{1} S$, listed in order of increasing energy.

The crystal field splits the $3 \mathrm{~d}$ levels. In the case of an octahedral field the atomic $3 \mathrm{~d}$ orbitals are split into three degenerate $t_{2} g$ orbitals $(x y, y z$ and $z x)$ and two degenerate $e_{\mathrm{g}}$ orbitals $\left(z^{2}\right.$ and $\left.x^{2}-y^{2}\right)$ separated by an energy 10Dq. These degeneracies are further removed in lower symmetries by inclusion of more crystal field parameters. For the distorted octahedron for the spatial structure of $\mathrm{NiF}_{2}$ the corresponding symmetry is tetragonal, and one has to introduce the parameters $D s$ and $D t$ [21]. In this work the program CTM XAS [21] was used to calculate the $\mathrm{x}$-ray absorption spectrum of $\mathrm{NiF}_{2}$ at the nickel $\mathrm{L}_{2,3}$ edges. 
Table I: Atomic multiplet, crystal field and charge transfer parameters (in eV) used in the XAS calculation. For the RIXS calculation the same crystal field parameters were used. For the meaning of the parameters see the text.

\begin{tabular}{lllllllll}
\hline Parameter & $10 D q$ & $D t$ & $D s$ & $\Gamma_{2 p}$ & $U$ & $V_{p d}$ & $\Delta$ & $T$ \\
& 1.0 & 0.03 & 0.12 & 0.24 & 5.5 & 6.5 & 7.0 & 1.0 \\
\hline
\end{tabular}

Charge transfer effects can also be included in the ionic calculation [10, $11,21]$. In this case the program also considers a configuration in which a ligand electron $\left(\mathrm{F}-2 \mathrm{p}\right.$ in $\left.\mathrm{NiF}_{2}\right)$ is transferred to the metal. One denotes as $3 \mathrm{~d}^{9} \mathrm{~L}$ the state with an extra electron in the $\mathrm{Ni} 3 \mathrm{~d}$ subshell, and a ligand hole denoted by $L$. After $x$-ray absorption this configuration is excited into the $2 p^{5} 3 d^{10} L$ state. The calculation allows interaction of the original ionic configurations and these charge transfer configurations through the inclusion of new, adjustable parameters [21]. These are the Hubbard energy $U$, which is the energy necessary for a $\mathrm{Ni} 3 d$ electron to move from one metal site to another; the nickel $2 p-3 \mathrm{~d}$ core hole potential $V_{\mathrm{p}} d$ that represents the shift in energy levels introduced by the $2 p$ hole in the metal, the charge transfer energy $\Delta$, which is the energy needed to move the F $2 p$ electron into the $\mathrm{Ni}$ ion, and the nickel site to nickel site hopping energy $T$. In this paper we present two $\mathrm{Ni}^{2+}$ $\mathrm{x}$-ray absorption spectra. One was calculated using only the crystal field part of the program, and and for the other we also included charge transfer effects. The parameters used in the calculation are shown in Table I.

The RIXS process is described by the Kramers-Heisenberg equation

$$
\sigma\left(v_{1}, v_{2}\right) \propto \sum_{f}\left|\sum_{i} \frac{<f\left|\hat{\varepsilon}_{2} \cdot \vec{r}\right| i><i\left|\hat{\varepsilon}_{1} \cdot \vec{r}\right| g>}{E_{i}-E_{g}-h v_{1}-i \Gamma_{2 p} / 2}\right|^{2} \delta\left(E_{f}-E_{i}-h\left(v_{1}-v_{2}\right)\right)
$$

In this formula $|g>| i>$, and $\mid f>$ are the wavefunctions of the initial, intermediate and final states of the system, with energies $E_{\mathrm{g}}, E_{\mathrm{i}}$ and $E_{\mathrm{f}}$, respectively; $\hat{\varepsilon}_{1} \cdot \vec{r}$ and $\hat{\varepsilon}_{2} \cdot \vec{r}$ are the electric dipole transition operators; and $\Gamma_{2 \mathrm{p}}$ is the $2 p$ core hole width. The output from the crystal field program, without any charge transfer effects, provides the values of the transition matrix elements and also for the state energies. This information was used to calculate $\mathrm{Ni}^{2+}$ RIXS spectra for all values of the incoming photon energy used in the experiment. For the RIXS calculation we used the crystal field parameters given in Table I.

\section{B. Density of states calculation}

To perform the electronic structure calculations, the WIEN2k code was used [2], which is based on the Density Functional Theory. This code is an all-
Atomic Multiplet and Charge

Transfer Effects in the Resonant Inelastic X-Ray Scattering (RIXS) Spectra at the Nickel $\mathrm{L}_{2,3}$ Edge of $\mathrm{NiF}_{2}$ 
Jiménez-Mier, J Olalde-Velasco, $\mathrm{P}$ de la Mora, $\mathrm{P}$ Yang, W -L Denlinger, $\mathrm{J}$

electron scheme based on the full-potential Linearized Augmented Plane Wave + local orbitals method. The band gaps calculated using the Kohn-Sham eigenvalues are known to be underestimated [8], an issue that can be fixed by using the GW approximation or a hybrid functional. However, these would be quite expensive, and an alternative solution incorporating the Becke-Johnson potential (BJ) [1] was used in this work. This potential is less demanding in computer time and it shows an agreement of the same order with the experiment as that obtained with the GW approximation, for wide band gap insulators, sp semiconductors and strongly correlated $3 \mathrm{~d}$ transition metal oxides $[1,22]$. In considering the exchange-correlation interactions, the generalized gradient approximation of Perdew, Burke and Ernzerhof (PBE) [20] was used, and it was then substituted after convergence, by the Becke-Johnson potential. For the number of plane waves used, the considered criterion was $R_{\min }^{M T} \times K^{\max }=$ 7 , where $R^{M T}$ is the Muffin-Tin radius and $K^{\max }$ is the maximum value of the $\mathrm{k}$ plane waves. Within the code used, $R_{\min }^{M T} \times K^{\max }$ is a measure of the quality of the basis set, and $1000 \mathrm{k}$-points were used.

\section{EXPERIMENT}

The experiment took place at beamline 8.0.1 of The Advanced Light Source in Berkeley. The experimental x-ray absorption spectrum was obtained by recording the sample current as the photon energy of the incoming $\mathrm{x}$-rays was scanned. Resonant x-ray emission spectra were recorded in a soft x-ray fluorescence spectrometer, which is a grazing incidence instrument with a fixed entrance slit and a position sensitive area detector [13]. Emission spectra were obtained at selected values of the incoming photon energy. Fresh, commercially available polycrystalline $\mathrm{NiF}_{2}$ from Sigma Aldrich with nominal purity greater than $99.9 \%$ was prepared inside a $\mathrm{N}_{2}$ flow bag and introduced inside the UHV experimental chamber. All spectra presented in this work were recorded at room temperature.

\section{RESULTS AND DISCUSSION}

The x-ray absorption spectrum at the nickel $\mathrm{L}_{2,3}$ edge of $\mathrm{NiF}_{2}$ is shown in Fig. 1 . The features around $850 \mathrm{eV}$ result from production of a $2 \mathrm{p}_{3 / 2}$ core hole and define the $\mathrm{L}_{3}$ edge. The smaller features beginning at $867 \mathrm{eV}$ correspond to the $\mathrm{L}_{2}$ edge, which corresponds to excitation of a $2 \mathrm{p}_{1 / 2}$ electron. A straight line background was subtracted from the raw spectrum to make the comparison with the multiplet calculation. The labels give the energies of the incoming photon that were used for the RIXS spectra. Results of two calculations are presented. 


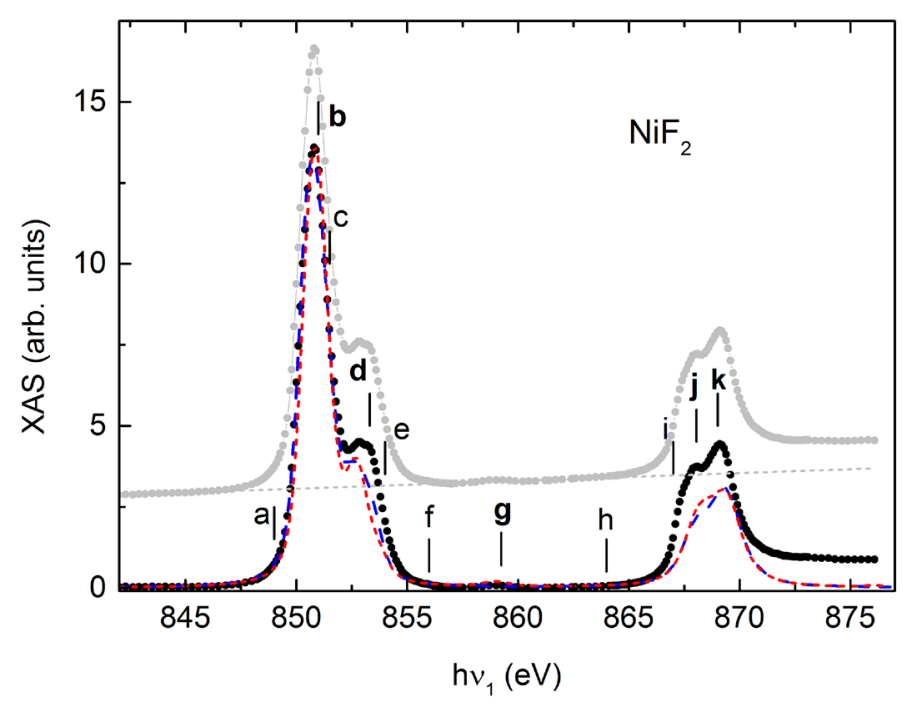

Figure 1: X-Ray absorption spectrum at the $\mathrm{Ni}_{2,3}$ edge of $\mathrm{NiF}_{2}$. Gray dots: raw spectrum. Black dots: spectrum after subtraction of a straight line background (dashed gray line). The continuous lines are the results of the multiplet calculation. Dashed blue with crystal field only. Red: with charge transfer effects. Labels a through k give the excitation energies that were used to record RIXS spectra.

In the first one (dashed blue line) only crystal field effects were included. For the second calculation charge transfer effects were also considered. The main difference between the two calculations is the presence of a small, broad peak at energy ( $\mathrm{g}$ ) that can therefore be identified as a charge transfer peak.

The nickel RIXS spectra are dominated by peaks resulting in decay into excited states of the metal $3 \mathrm{~d}^{8}$ ground configuration. The energy of these peaks puts them close to the $\mathrm{L}_{3}$ edge in absorption. The outgoing photons with energies above the $\mathrm{L}_{3}$ edge are efficiently absorbed by the sample and therefore the spectra must be corrected for this self-absorption effect. Figure 2 illustrates this effect for the emission spectrum obtained with excitation energy (d). There is a clear overlap between the raw emission spectrum and the absorption spectrum. Self-absorption results in a reduction of the intensity of the main emission peaks $y$ and $z$. Once the self-absorption correction is made [14] these two peaks grow in intensity. All emission spectra presented in this article were corrected for self-absorption. Figure 2 also serves to introduce the features present in the RIXS spectra. Elastic emission E corresponds to an outgoing photon with the same energy of the incoming photon. This means that no energy was absorbed by the sample, and therefore the final state of the
Atomic Multiplet and Charge

Transfer Effects in the Resonant Inelastic X-Ray Scattering (RIXS) Spectra at the Nickel $\mathrm{L}_{2,3}$ Edge of $\mathrm{NiF}_{2}$ 
Jiménez-Mier, J

Olalde-Velasco, $\mathrm{P}$

de la Mora, $\mathrm{P}$

Yang, W -L

Denlinger, $\mathrm{J}$

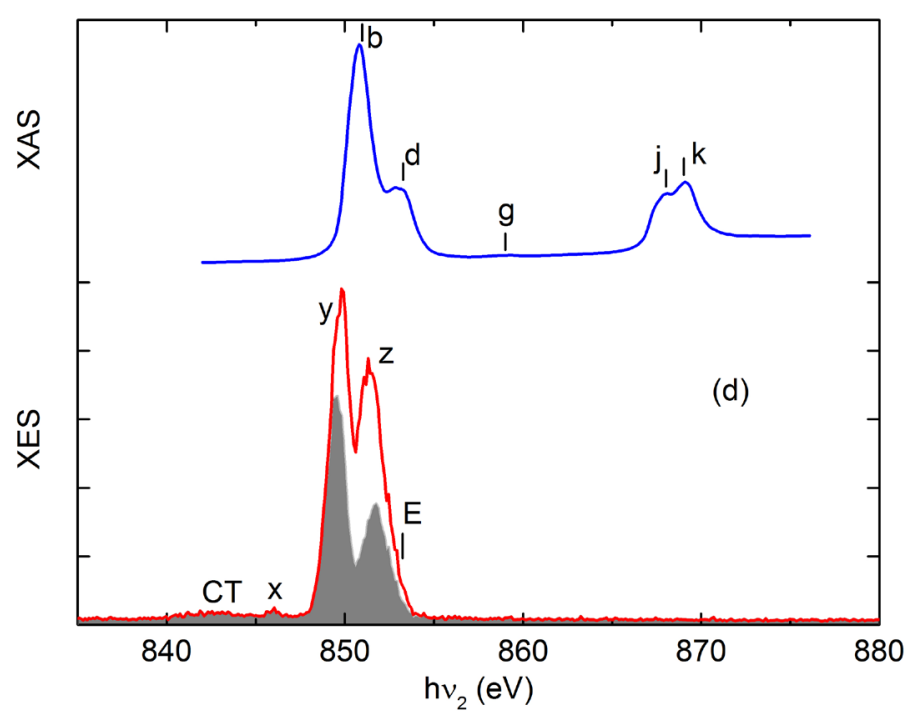

Figure 2: Example of self-absorption effects in an emission spectrum. The shaded gray is the raw spectrum and the red lines give the corrected spectrum. Bottom: RIXS spectrum recorded with an excitation energy (d). Top: XAS spectrum. The d-d excitations are indicated by $\mathrm{x}, \mathrm{y}$ and $\mathrm{z}$. E gives the position of the expected elastic peak. CT is the charge transfer peak.

scattering process is the ground state of the nickel ion. The spectrum shown in Fig. 2 has a very small elastic peak. This indicates that decay proceeds mainly into excited states of the $3 \mathrm{~d}^{8}$ configuration. This results in emission peaks $\mathrm{x}, \mathrm{y}$ and $\mathrm{z}$. This suppression of elastic emission is common to all the nickel RIXS spectra of $\mathrm{NiF}_{2}$. Another feature present in most of the spectra is the broad peak indicated by $\mathrm{CT}$ in Fig. 2. This results from decay leading to production of the $3 \mathrm{~d}^{9} \mathrm{~L}$ charge transfer state with an energy loss centered at $10.2 \mathrm{eV}$.

A comparison between experiment and the multiplet calculation for several representative emission spectra is made in Fig. 3. All experimental spectra have peaks resulting from decay into d-excited states, a small, charge transfer peak that stays $10.2 \mathrm{eV}$ below the position of the expected elastic peak. The spectra recorded at the $\mathrm{L}_{2}$ edge also show a strong normal emission peak (N). The agreement between experiment and theory is better for the spectra recorded at the $\mathrm{L}_{3}$ edge, for which the calculation give relative intensities close to the observed ones. The spectra obtained with excitation energies (j) and (k) are also dominated by the d-excited states. However, the experimental peaks are significantly broadened, which is an indication of the fast decay of the 


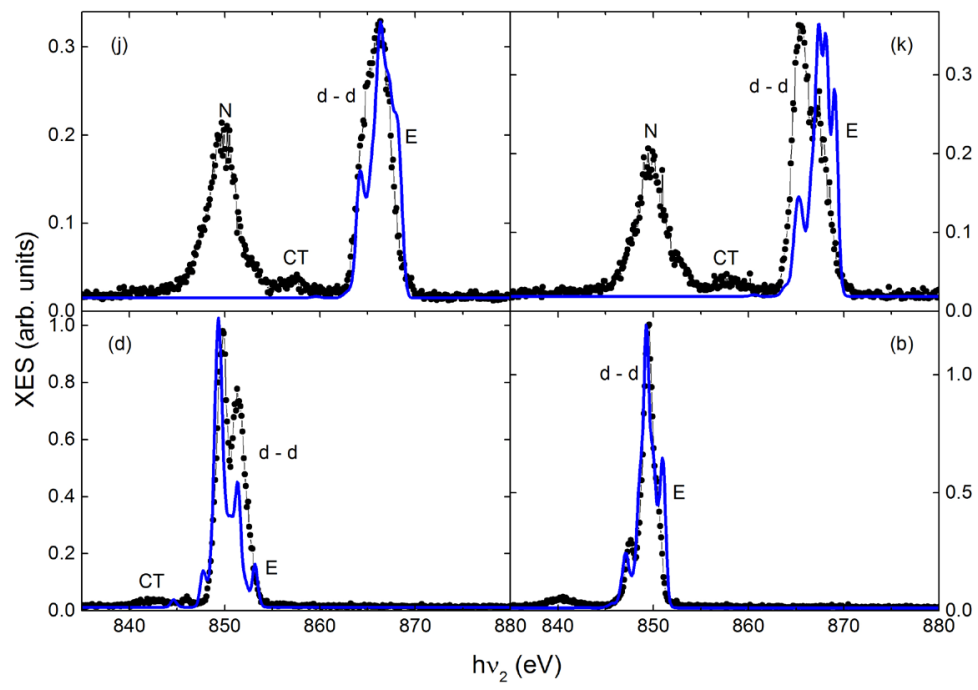

Figure 3: RIXS spectra recorded at excitation energies (b), (d), (j) and (k). Dots: experimental data. Blue line: calculated spectra. N: normal emission. CT: charge transfer emission. E: expected position of the elastic peak.

$2 \mathrm{p}_{1 / 2}$ hole by a non-radiative Coster-Kronig process. The agreement with the calculation is better for spectrum $(\mathrm{j})$, but for $(\mathrm{k})$ the calculation gives most of the intensity to the lower d-excited states while the peaks around $865 \mathrm{eV}$, with larger energy losses, have most of the intensity in the experimental data.

Figure 4 shows the emission spectrum recorded at the excitation energy (g). Recall that this is the only feature in the absorption spectrum that is not predicted by the simple crystal field calculation but requires the inclusion of charge transfer effects. The raw spectrum is given by the shaded area, and the spectrum obtained after the self-absorption correction is indicated by the red line. This spectrum has two separated features. A broad hump between 857 and $860 \mathrm{eV}$ is the one that corresponds to the suppressed elastic emission and to d-excited states. The structure between 845 and $852 \mathrm{eV}$ is composed of two peaks. There is a broad normal emission peak centered at $850 \mathrm{eV}$ that results from decay after excitation of the 2 p electron into the continuum. This therefore corresponds to decay from the $2 \mathrm{p}^{5} 3 \mathrm{~d}^{8}$ configuration of $\mathrm{Ni}^{3+}$. The other broad peak is centered at $848 \mathrm{eV}$, and results from decay of the resonantly produced charge transfer state of $\mathrm{Ni}^{2+}$.

These results are nicely rounded with a comparison between experiment and the density of states calculation. The comparison is made in Fig. 5. The
Atomic Multiplet and Charge

Transfer Effects in the Resonant Inelastic X-Ray Scattering (RIXS) Spectra at the Nickel $\mathrm{L}_{2,3}$ Edge of $\mathrm{NiF}_{2}$ 
Jiménez-Mier, J Olalde-Velasco, $\mathrm{P}$ de la Mora, $\mathrm{P}$ Yang, W -L

Denlinger, $\mathrm{J}$

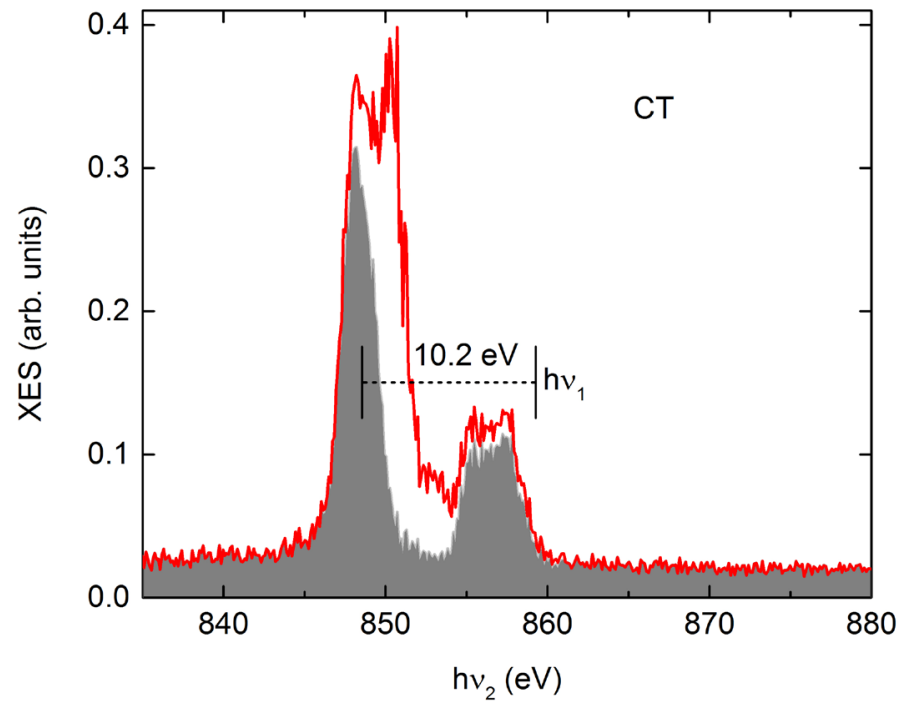

Figure 4: RIXS spectrum for the charge transfer excitation energy (g). Shaded gray area: raw spectrum. Red line: spectrum corrected for self-absorption.

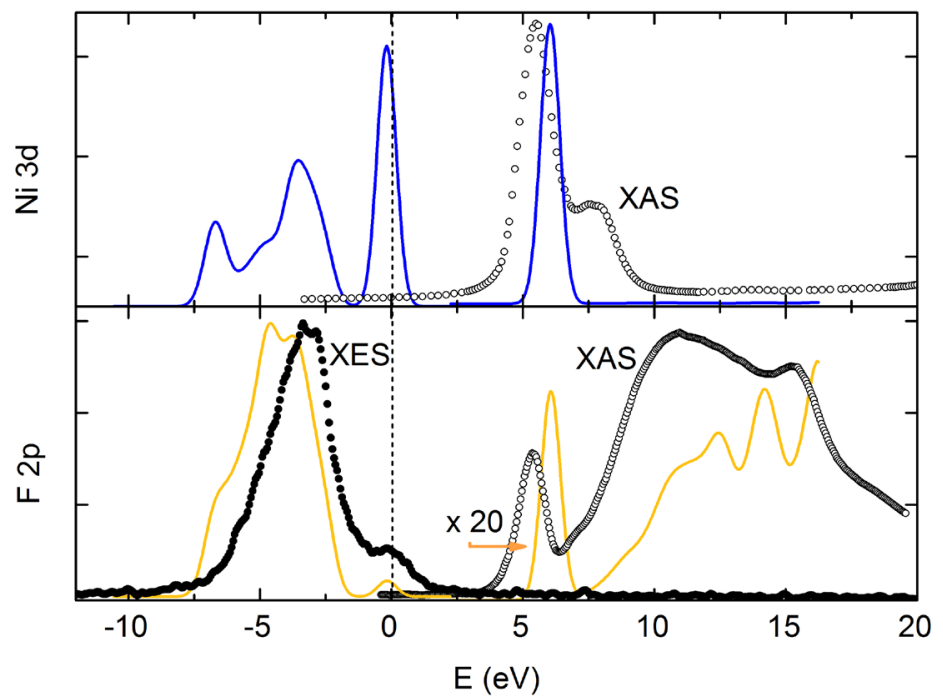

Figure 5: Comparison between experiment and the calculated projected density of states (DOS). Bottom: experimental XAS (open circles) and XES (dots) spectra at the fluorine K-edge. The continuous yellow line is the calculated F 2p DOS. The conduction band data was multiplied by a a factor of 20 . Top: experimental $\mathrm{Ni} \mathrm{L}_{3}$ absorption (open circles). The continuous blue line is the calculated Ni 3d DOS. 
experimental data shown here are the absorption spectra at the $\mathrm{L}_{3}$ edge in nickel, and the fluorine absorption and emission spectra at the fluorine $\mathrm{K}$ edge. The figure also shows the result of a convolution of projected density of states with a Gaussian window whose width is $0.5 \mathrm{eV}$. This figure illustrates the information that can be directly obtained from XAS and XES spectra. Both absorption spectra are aligned at the peak at $5.5 \mathrm{eV}$. The DOS calculation indicates that this feature corresponds to excitation into a narrow conduction sub-band predominantly Ni $3 \mathrm{~d}$ in character. The fluorine emission spectrum is then given by decay of the valence band which is mainly of $F 2 p$ character. The calculation clearly overestimates the band gap, but confirms this band assignment, and is also consistent with the ZSA theory [18]. It is really remarkable that the shape of the fluorine emission spectrum is in very good agreement with the calculated $\mathrm{F} 2 \mathrm{p}$ density of states, once the theoretical curve is shifted upwards. It gives an accurate prediction of the intensity ratio between the fluorine $2 \mathrm{p}$ and nickel $3 \mathrm{~d}$ DOS ratios projected at the fluorine atom.

\section{CONCLUSIONS}

The results presented in this work show that $\mathrm{x}$-ray spectroscopies combined with a theoretical analysis can be very useful in the study of the electronic structure of transition metal compounds. These techniques were used to interpret $\mathrm{x}$-ray absorption and emission at the fluorine $\mathrm{K}$-edge and the nickel $\mathrm{L}_{2,3}$ edges of the most ionic nickel compound, namely $\mathrm{NiF}_{2}$. Nickel $\mathrm{L}_{2,3}$ RIXS spectra are dominated by $\mathrm{d}-\mathrm{d}$ excitations of the $3 \mathrm{~d}^{8}$ configuration, but there is also indication of charge transfer emission. Fluorine x-ray absorption and emission are well explained by a density of states calculation that also shows strong hybridization between fluorine $2 \mathrm{p}$ states and nickel $3 \mathrm{~d}$ states at the metal site. By a systematic approach combinig atomic like charge transfer mutlitplet calculations to describe the XAS and RIXS spectra of $\mathrm{Ni}^{2+}$ at the $\mathrm{L}_{2,3}$ edges and a band approach using LDA+U calculations to describe the F K XAS and XES spectra, we reach a comprehensive understanding of the electronic structure of $\mathrm{NiF}_{2}$.

\section{ACKNOWLEDGMENTS}

This research used resources of the Advanced Light Source, which is a DOE Office of Science User Facility under contract no. DE-AC02-05CH11231.

\section{REFERENCE}

[1] Becke, A.D. \& Johnson, E.R. (2006) A simple effective potential for exchange. J. Chem. Phys. 24, \#221101.
Atomic Multiplet and Charge

Transfer Effects in the Resonant Inelastic X-Ray Scattering (RIXS) Spectra at the Nickel $\mathrm{L}_{2,3}$ Edge of 
Jiménez-Mier, $\mathrm{J}$ Olalde-Velasco, $\mathrm{P}$ de la Mora, $\mathrm{P}$ Yang, W -L Denlinger, J
[2] Blaha, P. et al. WIEN2k an Augmented Plane Wave Plus Local Orbital Program for Calculating Crystal Properties, Vienna University of Technology, Vienna, 2001.

[3] Chiuzb aian, S. G. et al. (2005) Localized Electronic Excitations in NiO Studied with Resonant Inelastic X-Ray Scattering at the Ni M Threshold: Evidence of Spin Flip. Phys. Rev. Lett. 95, \#197402

[4] Cowan, R.D. (1981). The Theory of Atomic Structure and Spectra, Berkeley: University of California Press.

[5] Dagotto, E. (2005) Complexity in Strongly Correlated Electronic Systems, Science 309, 257-262.

[6] Dufek, P., Schwarz, K. \& Blaha, P. (1993) Electronic structure of $\mathrm{MnF}_{2}$ and $\mathrm{NiF}_{2}$, Phys. Rev. B 48, 12672-12681.

[7] Ghiringhelli, G. et al. (2009) Observation of Two Nondispersive Magnetic Excitations in NiO by Resonant Inelastic Soft-X-Ray Scattering, Phys. Rev. Lett. 102, \#027401.

[8] Godby, R., Schlu“ter, M. \& Sham, L. (1986) Accurate Exchange-Correlation Potential for Silicon and Its Discontinuity on Addition of an Electron. Phys. Rev. Lett. 56, 2415-2418.

[9] de Groot, F. \& Kotani, A. (2008) Core Level Spectroscopy of Solids, Boca Raton, Fl, CRC Press.

[10] de Groot, F. (2001) High-Resolution X-ray Emission and X-ray Absorption Spectroscopy. Chemistry Review 101, 1779-1808.

[11] de Groot, F. (2005) Multiplet effects in X-ray spectroscopy. Coordination Chemistry Reviews 249, 31-63.

[12] Imada, M., Fujimori, A. \& Tokura, Y. (1998) Metal-insulator transitions, Rev. Mod. Phys. 70, 1039-1263.

[13] Jia, J.J. et al. (1995) First experimental results from IBM/TENN/TULANE/ LLNL/LBL undulator beamline at the advanced light source. Rev. Sci. Instrum. 66, 1394-1397.

[14] Jiménez-Mier, J., Ederer, D.L. \& Schuler, T. (2005) X-ray Raman scattering at the manganese $\mathrm{L}$ edge of $\mathrm{MnF}_{2}$ : Valence emission of $\mathrm{Mn}^{2+}$. Phys. Rev. A 72, \#022502.

[15] van der Laan, G. et al. (1986) Comparison of X-ray absorption with X-ray photoemission of nickel diahlides and NiO, Phys. Rev. B, 33, 4253-4263.

[16] Lee, D. H. et al. (2012) Conversion mechanism of nickel fluoride and NiO-doped nickel fluoride in Li ion batteries. Electrochimica Acta 59, 213-221.

[17] Lee, D.H. et al. (2014) Understanding improved electrochemical properties of $\mathrm{NiO}$-doped $\mathrm{NiF}_{2}-\mathrm{C}$ composite conversion materials by X-ray absorption spectroscopy and pair distribution function analysis, Phys. Chem. Chem. Phys. 16, 3095-3102.

[18] Olalde-Velasco, P. et al. (2011) Direct probe of Mott-Hubbard to charge-transfer insulator transition and electronic structure evolution in transition-metal systems, Phys. Rev B. 83, \#241102(R). 
[19] Olalde-Velasco, P., Jiménez-Mier, J., Denlinger, J., \& Yang, W.-L. (2013) Atomic multiplets at the $\mathrm{L}_{2,3}$ edge of $3 \mathrm{~d}$ transition metals and the ligand $\mathrm{K}$ edge in X-ray absorption spectroscopy of ionic systems, Phys. Rev. B 87, \#245136.

[20] Perdew J.P., Burke, K \& Ernzerhof, M. (1996) Generalized Gradient Approximation Made Simple. Phys. Rev. Lett. 77, 3865-3868.

[21] Stavitski, E. \& de Groot, F.M.F. (2008) The CTM4XAS program for EELS and XAS spectral shape analysis of transition metal L edges. Micron 41, 687-694.

[22] Tran,F.\& Blaha,P.(2009) Accurate Band Gaps of Semiconductors and Insulators with a Semilocal Exchange-Correlation Potential. Phys. Rev. Lett. 102, \#226401.

[23] Yang, L., Luo, W. \& Chen, G.-Z. (2013) In Situ Synthesis of Ni(o) Catalysts Derived from Nickel Halides for Hydrolytic Dehy-drogenation of Ammonia Borane. Catalysis Letters, 143, 873-880.

[24] Yang, Y. et al. (2014) Flexible Three-Dimensional Nanoporous Metal-Based Energy Devices. J. Am. Chem. Soc. 136, 6187-6190.

[25] Zaanen, J., Sawatzky, G.A. \& Allen, J.W. (1985) Band Gaps and Electronic Structure of Transition-Metal Compounds, Phys. Rev. Lett. 55, 418-421.

[26] Zhang, H., Zhou, Y,-N., Sun, Q., \& Fu, Z,-W. (2008) Nanostructured nickel fluoride thin film as a new Li storage material, Solid State Sciences 10, 1166-1172.
Atomic Multiplet and Charge

Transfer Effects in the Resonant Inelastic X-Ray Scattering (RIXS) Spectra at the Nickel $\mathrm{L}_{2,3}$ Edge of $\mathrm{NiF}_{2}$

$\mathrm{NiF}_{2}$

